\title{
Multiple-Attribute Decision-Making Problem Using TOPSIS and Choquet Integral with Hesitant Fuzzy Number Information
}

\author{
Harish Garg $\mathbb{D}^{1},{ }^{1}$ Abazar Keikha, ${ }^{2}$ and Hassan Mishmast $\mathrm{Nehi}^{3}$ \\ ${ }^{1}$ School of Mathematics, Thapar Institute of Engineering and Technology, Deemed University, Patiala 147004, Punjab, India \\ ${ }^{2}$ Department of Mathematics, Velayat University, Iranshahr, Iran \\ ${ }^{3}$ Department of Mathematics, University of Sistan and Baluchestan, Zahedan, Iran \\ Correspondence should be addressed to Harish Garg; harishg58iitr@gmail.com
}

Received 7 July 2020; Revised 7 September 2020; Accepted 16 September 2020; Published 17 October 2020

Academic Editor: S. A. Edalatpanah

Copyright ( $\odot 2020$ Harish Garg et al. This is an open access article distributed under the Creative Commons Attribution License, which permits unrestricted use, distribution, and reproduction in any medium, provided the original work is properly cited.

\begin{abstract}
The paper aims are to present a method to solve the multiple-attribute decision-making (MADM) problems under the hesitant fuzzy set environment. In MADM problems, the information collection, aggregation, and the measure phases are crucial to direct the problem. However, to handle the uncertainties in the collection data, a hesitant fuzzy number is one of the most prominent ways to express uncertain and vague information in terms of different discrete numbers rather than a single crisp number. Additionally, to aggregate and to rank the collective numbers, a TOPSIS ("Technique for Order of Preference by Similarity to Ideal Solution") and the Choquet integral (CI) are the useful tools. Keeping all these features, in the present paper, we combine the TOPSIS and CI methods for hesitant fuzzy information and hence present a method named as TOPSIS-CI to address the MADM problems. The presented method has been described with a numerical example. Finally, the validity of the stated method as well as a comparative analysis with the existing methods is addressed in detail.
\end{abstract}

\section{Introduction}

Decision-making plays a vital role in the practical life activities of human beings as it refers to a process that lays out all the options according to the assessment data of the decision makers and then selects the excellent one, mostly happening in our everyday lives. In the early era of social development, decision makers utilized the real numbers as a rule to offer their assessment information. As the multiattribute decision-making (MADM) problems are becoming complex, the experts cannot give exact real numbers to assess the alternatives. The ambiguities and imprecision of human judgments highlighted the deficiency of the crisp set theory. To handle it, a theory of fuzzy set (FS) [1] has been initiated for uncertain knowledge that permits the experts to describe their satisfaction level (membership degree). Since its appearance, many scholars have addressed the applications of the FS to different fields [2-7]. In the FS theory, a single membership degree has been assigned to each element. However, when there is any kind of hesitation during the evaluation process by an expert, a more generalized concept of the FS is much needed. For it, a theory of the hesitant fuzzy set (HFS) [4] came into the picture which allows the membership grades presuming a set of possible values instead of a single one. In an HFS, the set of membership degrees assigned to each member is called a hesitant fuzzy element (HFE). Later on, Torra and Narukawa [8] presented a relationship between the HFS and other extended FSs and found that the intuitionistic fuzzy set [9] appeared to be an envelope of HFS. Deepak et al. [10] presented a topological structure involving the HFSs.

Since its appearance, many scholars have worked on it and presented several applications and algorithms to solve the MADM problems. In general, there are three kinds of phases for solving any MADM. The first phase is information collection in which rating corresponding to the available alternatives is accessed under the different attributes using the HFE features. The second phase is to obtain the collective information of each alternative. For it, information measures or an aggregation operator $(\mathrm{AO})$ are 
much widely and successfully used. The last phase of the MADM approach is to rank the given numbers based on some defuzzified values.

In the last decades, extensive theoretical studies of numerous MADM problems under the HFS environment have been noticed. For instance, Xu and Xia [11] presented some distance measures on HFSs. However, Xu and Xia [7] presented distance and correlation measures to solve the MADM problem under the HFS environment. Verma and Sharma [12] presented some operations over HFSs. Liao et al. [13] presented the multiplicative consistency of the hesitant fuzzy preference relation and applied them to solve the group decision-making problems. Also, in it, they presented the score and variance functions of HFSs to compare the HFSs. Also, Farhadinia [14] presented a series of score functions for HFSs. Liao et al. [15] presented a power average AOs to aggregate the HFS information. Zhang [16] presented the power aggregation operators for HFSs to solve the MADM problems. Lalotra and Singh [17] presented a concept of hesitant fuzzy knowledge measure and investigate hesitant fuzzy entropy to solve the problems. The other powerful tools used for the processing the information by the various researchers are TOPSIS (Technique for Order of Preference by Similarity to Ideal Solution), VIKOR (VIseKriterijumska Optimizacija I Kompromisno Resenje), TODIM (an acronym in Portuguese of interactive and multicriteria decisionmaking), etc. Under these, Zhang and Wei [18] extended the standard VIKOR method to the HFS information and applied them to solve the MADM problem. Xu and Zhang [19] presented a hesitant fuzzy MADM based on the TOPSIS method. Tong and Yu [20] presented a MADM algorithm based on distance and correlation coefficient with HFE information. $\mathrm{Xu}$ and $\mathrm{Xia}$ [21] presented entropy and crossentropy measure-based algorithm to solve the MADM problems. Some recent approaches regarding decisionmaking problems with TOPSIS approaches can be found in [22-29] and their corresponding references.

In terms of the AOs, Xia and $\mathrm{Xu}$ [30] presented weighted operators for hesitant fuzzy information. Zhang and $\mathrm{Xu}$ [31] presented a TODIM analysis to solve the decision-making problem under the hesitant fuzzy information. Wei [32] developed some prioritized AOs to deal with MADM problems under a HFS environment. Liao and $\mathrm{Xu}$ [33] developed a VIKOR method for solving MADM under HFS environment. He [34] presented a Dombi t-norm-based operational laws for HFSs and their corresponding AOs for solving the MADM problems. Wei et al. [35] and Demirel et al. [36] developed the Choquet integral- (CI-) based AOs to solve the hesitant fuzzy MADM problems. Zhu et al. [37] presented the geometric Bonferroni mean AOs for HFS. Xu and Zhou [38] implemented hesitant probabilistic fuzzy operations and used them to solve group decision-making problems. Zhang and Wu [39] presented a weighted HFS and their AOs based on Archimedean t-norm operations. Liao and $\mathrm{Xu}$ [40] presented the extended hesitant fuzzyweighted AOs to solve the MADM problems. Wei et al. [41] presented an arithmetic and geometric AOs for hesitant fuzzy linguistic information. Tan et al. [42] presented Hamacher t-norm-based operational rules for HFSs.
From the above investigation, it has been observed that the HFS is a powerful tool to solve the decision-making problems and hence widely used in the literature. Recently, Ranjbar et al. [43] presented a new extension of the fuzzy numbers to the hesitant fuzzy numbers (HFNs). Also, they defined the $(\alpha, k)$ cuts of HFNs, binary operations, and a relationship for comparing the two HFNs. In general, a HFN is a special case of a HFS of the real line. Deli [25] presented a generalized trapezoidal hesitant fuzzy (GTHF) numbers and hence introduced some distance measures including Hamming, Euclidean, and Hausdorff. Based on these measures, a TOPSIS approach is presented to solve the MADM problems of GTHF numbers. As we know, the elements of decision matrix $D=(d)$ in any MADM problem are the direct evaluations of the expert from alternatives against all attributes. Due to some of the details that may have been overlooked by expert and complexities of problems, we generally use the self-assessment process to form the decision matrix. In today's decision-making, self-assessment is one of the important factors for evaluation in all dimensions from individual to social, educational, organizational, corporate, income, tax, etc. The self-assessment process is important because it is the most appropriate way to measure the activities performed by a unit over a period of time. So who is better to evaluate it than those who have been involved in the activity? For example, taxpayers in self-declaration forms state amounts as income that may need to be assessed by a tax officer. The government also plans for the future by relying on amounts set by taxpayers, which may not be accurate or the actual amount may be less/more than stated. Usually, predesigned forms are provided to the evaluator, and she/he will eventually earn points by filling out these forms and uploading related documents of each score. What is at stake are covert motives that, for whatever reason such as earning higher scores, make it necessary to review and verify the results, which can be done by the evaluator or higher level managers. It should be noted that self-assessment is not the only context in which we are confronted with values that may not be accurate. In astronomy, medicine, economics, etc., there is preproduced numerical information that researchers are skeptical about, and in future decisions, it is necessary to use this data along with researchers' opinions. Therefore, it is necessary to handle these expert crisp values in a set of values from $[0,1]$ and form hesitant fuzzy elements (HFEs) $(h)$. Hence, the earlier obtained decision matrix $D=$ (d) is converted to hesitant decision matrix $\overparen{H D}=(\langle d, h\rangle)$ with HFNs. Since, the existing work is only based on the hesitant fuzzy information $D=(d)$ which completely ignores the part of the self-assessment process. Thus, there is a need to design an efficient approach by using the features of the HFNs to solve the MADM problems.

This work aims to fulfill this gap by directing an approach based on the TOPSIS method for solving the MADM problems under the HFN environment. The TOPSIS method, initially introduced by Hwang and Yoon [44], is one of the most widely used ones and plays an important role in the hesitant fuzzy decision-making process [18, 19, 26, 45]. Further, in a MADM problem, an interrelationship between the attribute information is also important to get the fair decision. To handle it, a Choquet integral (CI) operator has been utilized to address it. The benefit of using CI during the 
process is that it practices fuzzy measures during its estimates and also reflects the intercommunication between all becoming pairs of attributes. In the proposed method, the given alternatives are evaluated under the set of the HFNs including the self-assessment process. In it, an uncertainty which is presented in the data is handled with the help of the hesitant fuzzy information which consists of two parts: real and membership parts. Also, the Hamming and Euclidean distances of such HFNs are also defined. Based on these measures, we stated the TOPSIS method in which the relative positive and negative strengths of the alternatives are aggregated with the help of CI and hence presented an algorithm named as TOPSIS-CI. Finally, the "satisfaction value" of the alternative is determined with the closeness degree. The presented approach has been demonstrated with a numerical example and performs some validity test to examine their performance.

The rest paper is summarized as follows. Section 2 presents some basic concepts on HFSs. In Section 3, a new TOPSIS method based on CI has been presented to address the MADM problems. In Section 4, a numerical example is presented to demonstrate the stated method. Finally, a conclusion is written in Section 5.

\section{Preliminaries}

In this section, some basic concepts related to HFSs are reviewed over the set $\mathscr{X}$.

Definition 1 (see [4]). A HFS $E$ on $\mathscr{X}$ is stated as

$$
E=\{\langle x, h(x)\rangle \mid x \in \mathscr{X}\},
$$

where $h$ is a function that belongs to a subset of $[0,1]$ and number of elements of $h(x) \subset[0,1]$ are finite and expressed as membership degrees of $x \in \mathscr{X}$ to the set $E$ [30]. Mathematically, it can be expressed as

$$
E=\underset{\gamma \in h}{\cup}\{\langle x, \gamma\rangle \mid x \in \mathscr{X}\}
$$

where $\gamma \in[0,1]$

Note 1. An element $h(x)=\left\{\gamma_{1}, \gamma_{2}, \ldots, \gamma_{n}\right\}$ where $\gamma_{i} \in[0,1](i=1, \ldots, n)$ is called hesitant fuzzy element (HFE).

Definition 2 (see $[13,30]$ ). For $\operatorname{HFE~} h$, mean (or score function), hesitant degree, and variance of its are, respectively, defined as

$$
\begin{aligned}
\bar{h}(x) & =S(h)=\left(\frac{1}{n}\right) \sum_{i=1}^{n} \gamma_{i} ; \\
\operatorname{Var}(h) & =\sqrt{\left(\frac{1}{n}\right) \sum_{i=1}^{n}\left(\gamma_{i}-\bar{h}(x)\right)^{2}} ; \\
\phi_{h(x)} & =\left(\frac{1}{n}\right) \sqrt{\sum_{\gamma_{i}, \gamma_{j}}\left(\gamma_{i}-\gamma_{j}\right)^{2}} .
\end{aligned}
$$

Definition 3 (see [13]). An order relation between two HFEs $h_{1}$ and $h_{2}$, denoted by $h_{1} \prec h_{2}$ holds if either of the conditions satisfy:

$$
\begin{aligned}
& \text { (1) } S\left(h_{1}\right)<S\left(h_{2}\right) \\
& \text { (2) } S\left(h_{1}\right)=S\left(h_{2}\right) \text { and } \operatorname{Var}\left(h_{1}\right)>\operatorname{Var}\left(h_{2}\right)
\end{aligned}
$$

For two different HFEs, their numbers are not necessarily of the same length, and this is debatable to convert them to the same size as adjusted HFEs. Let $h_{1}$ and $h_{2}$ be two HFEs, where $\left|h_{1}\right|=m$ and $\left|h_{2}\right|=n$ with $m<n$. To obtain adjusted HFEs, the length of $h_{1}$ must be upgraded to $n$ by adding $n-m$ elements to it. To this end, there exist many methods such as optimistic researchers proposed the maximum member of $h_{1}$, the pessimistic researchers considered its minimum element, the indifference ones considered the value of 0.5 [46], and it has recently been suggested that the power average of the available elements to be added $n-m$ times to the set $h_{1}$ [15].

Definition 4 (see [11]). Consider two adjusted HFEs $h_{1}$ and $h_{2}$. Let $h_{j(i)}$ be the $i$ th smallest value of $h_{j}$ and $l$ be the maximum length of the given HFEs, then

$$
d_{\mathrm{hnh}}\left(h_{1}, h_{2}\right)=\left(\frac{1}{l}\right) \sum_{i=1}^{l}\left|h_{1(i)}-h_{2(i)}\right|
$$

$$
\text { and } d_{\text {hne }}\left(h_{1}, h_{2}\right)=\sqrt{\left(\frac{1}{l}\right) \sum_{i=1}^{l}\left|h_{1(i)}-h_{2(i)}\right|^{2}} \text {. }
$$

are called hesitant normalized Hamming and Euclidean distances, respectively.

Definition 5 (see [13]). For a collection of HFEs $h_{j}(j=1,2, \ldots, n)$ and a positive real number $\lambda$, we have
(1) $h^{\lambda}=\left\{\left(h^{\sigma(t)}\right)^{\lambda} \mid t=1,2, \ldots, l\right\}$
(2) $\lambda h=\left\{1-\left(1-h^{\sigma(t)}\right)^{\lambda} \mid t=1,2, \ldots, l\right\}$
(3) $h_{1} \oplus h_{2}=\left\{h_{1}^{\sigma(t)}+h_{2}^{\sigma(t)}-h_{1}^{\sigma(t)} h_{2}^{\sigma(t)} \mid t=1,2, \ldots, l\right\}$
(4) $h_{1} \otimes h_{2}=\left\{h_{1}^{\sigma(t)} h_{2}^{\sigma(t)} \mid t=1,2, \ldots, l\right\}$
(5) $\oplus_{j=1}^{n} h_{j}=\left\{1-\prod_{j=1}^{n}\left(1-h_{j}^{\sigma(t)}\right) \mid t=1,2, \ldots, l\right\}$
(6) $\otimes_{j=1}^{n} h_{j}=\left\{\prod_{j=1}^{n} h_{j}^{\sigma(t)} \mid t=1,2, \ldots, l\right\}$

where $h_{j}^{\sigma(t)}$ is the $t$ th smallest value in $h_{j}$.

Definition 6 (see [13]). Let $h_{j}(j=1,2, \ldots, n)$ be a collection of HFEs, and $w_{j}>0$ with $\sum_{j=1}^{n} w_{j}=1$ be the weight vector of $h_{j}$, then we have the following:

(1) An adjusted hesitant fuzzy weighted average (AHFWA) operator is

$$
\begin{aligned}
& \operatorname{AHFWA}\left(h_{1}, h_{2}, \ldots, h_{n}\right)=\oplus_{j=1}^{n} w_{j} h_{j} \\
& =\left\{1-\prod_{j=1}^{n}\left(1-h_{j}^{\sigma(t)}\right)^{w_{j}} \mid t=1,2, \ldots, l\right\} .
\end{aligned}
$$


(2) An adjusted hesitant fuzzy weighted geometric (AHFWG) operator is

$$
\begin{gathered}
\operatorname{AHFWG}\left(h_{1}, h_{2}, \ldots, h_{n}\right)=\otimes_{j=1}^{n}\left(h_{j}\right)^{w_{j}} \\
=\left\{\prod_{j=1}^{n}\left(h_{j}^{\sigma(t)}\right)^{w_{j}} \mid t=1,2, \ldots, l\right\} .
\end{gathered}
$$

Note 2. When $w_{j}=(1 / n)$ for each $j$, then the AHFWA and AHFWG operators reduced to adjusted hesitant fuzzy average and geometric operator, labelled as AHFA and AHFG, respectively

In some real problems, such as self-assessment problems, option scores are obtained relative to each criterion through predesigned forms that are not fully accepted for any reason, and each score with a finite number of values between 0 and 1 , as the hesitation degrees of that score or DM' evaluation values of options, is accompanied. With the extension of applications of HFSs in the real world on the one hand and variety and complexity of real problems on the other hand, it was cleared that existing kind of HFSs cannot model all situations. For this reason, different types of hesitant fuzzy numbers (HFNs) with reference set $\mathbb{R}$, as the generalization of HFSs, have been proposed [25, 43].

Definition 7 (see [25]). Let $\widetilde{h}_{j}(j=1,2, \ldots, n)$ be a collection of fuzzy numbers with membership function $\mu_{h_{j}},(j=1,2, \ldots, n)$ such that $\cap_{j=1}^{n} \widetilde{h}_{j} \neq \phi$. Then,

$$
\tilde{h}=\left\{\left\langle x,\left\{\mu_{h_{1}}, \mu_{h_{2}}, \ldots, \mu_{h_{n}}\right\}\right\rangle, \quad x \in \mathbb{R}\right\} .
$$

is called a hesitant fuzzy number (HFN). Based on it, a generalized trapezoidal hesitant fuzzy number (GTHFN) is stated as

$$
A=\left\{\left\langle(a, b, c, d) ;\left\{\gamma_{i}: \gamma_{i} \in \gamma(x), i=1,2, \ldots, l\right\}\right\rangle\right\},
$$

where $a<b<c<d$ are real parameters, $\gamma(x)$ is a set of some values in $[0,1]$, and membership functions are defined as $l$ trapezoidal in $[0,1]$ with height $\gamma_{i}$.

\section{Proposed MADM Method}

In this section, we define the distance measure between the pairs of the HFNs and hence stated a TOPSIS algorithm based on CI to solve the MADM problems.

3.1. Distance Measure between HFNs. Let $a \in \mathbb{R}$ be a given assessment value which is obtained in any possible way and has degrees of uncertainty. The uncertainty is modeled via a HFE $h(a)$. Then, the ordered pair $\langle a, h(a)\rangle$ is called an HFN and diplayed by $\tilde{a}_{H}=\langle a, h(a)\rangle$, where $h(a)$ is a finite set of some values in $[0,1]$. The formal definition is given as follows.

Definition 8. Let $\mathscr{X}$ be the reference set and $a \in \mathbb{R}$, and a HFN $\widetilde{a}_{H}$ in the set of real numbers $\mathbb{R}$ is defined as

$$
\tilde{a}_{H}=\{\langle a, h(a)\rangle \mid a \in \mathscr{X}\}
$$

where $h(a)$ is a finite set of some values in $[0,1]$ and are considered as membership degrees of $a \in \mathscr{X}$. In this set, an HFN has two parts: real part and membership part. The elements of the second part are also interpreted as the satisfaction/hesitation degrees with the real part. Here, $0 \in h(a)$ is in the sense of complete rejection and $1 \in h(a)$ means the complete acceptance of the value $a$.

Definition 9. Let $\tilde{a}_{H}=\langle a, h(a)\rangle$ and $\tilde{b}_{H}=\langle b, h(b)\rangle$ be two HFNs and $\lambda>0$, then the following is obtained:

(1) $\tilde{a}_{H} \oplus \widetilde{b}_{H}=\langle a+b, h(a) \cup h(b)\rangle$, where $h(a) \cup h(b)=$ $\cup_{\gamma_{1} \in h(a), \gamma_{2} \in h(b)} \max \left\{\gamma_{1}, \gamma_{2}\right\}$

(2) $\tilde{a}_{H} \otimes \widetilde{b}_{H}=\langle a . b, h(a) \cap h(b)\rangle$, where $h(a) \cap h(b)=$ $\cup_{\gamma_{1} \in h(a), \gamma_{2} \in h(b)} \min \left\{\gamma_{1}, \gamma_{2}\right\}$

(3) $\lambda \tilde{a}_{H}=\langle\lambda a, h(a)\rangle$

(4) $\left(\widetilde{a}_{H}\right)^{\lambda}=\left\langle a^{\lambda}, h(a)\right\rangle$

Next, we state the Hamming distance $\left(d_{H}\right)$ and the Euclidean distance $\left(d_{E}\right)$ for the HFNs.

Definition 10. Consider two HFNs $\tilde{a}_{H}=\langle a, h(a)\rangle$ and $\tilde{b}_{H}=\langle b, h(b)\rangle$, where $a, b \in \mathbb{R}$, and $h(a)$ with cardinality $|h(a)|=k$ and $|h(b)|=l$. The distance measure in between these HFNs based on Hamming and Euclidean measures are stated as follows.

(1) Hamming distance:

$$
d_{H}\left(\tilde{a}_{H}, \tilde{b}_{H}\right)=\left(\frac{1}{1+k \times l}\right)\left(|a-b|+\sum_{\substack{\gamma_{i} \in h(a), \gamma_{j} \in h(b)}}\left|\gamma_{i}-\gamma_{j}\right|\right)
$$

(2) Euclidean distance:

$$
d_{E}\left(\tilde{a}_{H}, \tilde{b}_{H}\right)=\sqrt{\left(\frac{1}{1+k \times l}\right)\left(|a-b|^{2}+\sum_{\gamma_{i} \in h(a), \gamma_{j} \in h(b)}\left|\gamma_{i}-\gamma_{j}\right|^{2}\right)}
$$

Theorem 1. For three HFNs $\tilde{a}_{H}=\langle a, h(a)\rangle$, $\tilde{b}_{H}=\langle b, h(b)\rangle$, and $\widetilde{c}_{H}=\langle c, h(c)\rangle$, their proposed distance measures $d_{H}$ and $d_{E}$ as in Definition 10 must satisfy the following properties:
(1) $d_{E}\left(\widetilde{a}_{H}, \widetilde{b}_{H}\right)=d_{E}\left(\widetilde{b}_{H}, \tilde{a}_{H}\right)$, $d_{H}\left(\widetilde{a}_{H}, \vec{b}_{H}\right)=d_{H}\left(b_{H}, \widetilde{a}_{H}\right)$
(2) $d_{E}\left(\widetilde{a}_{H}, \tilde{a}_{H}\right)=0$ and $d_{H}\left(\widetilde{a}_{H}, \tilde{a}_{H}\right)=0$
(3) $d_{E}\left(\widetilde{a}_{H}, \widetilde{c}_{H}\right) \leq d_{E}\left(\widetilde{a}_{H}, \widetilde{b}_{H}\right)+d_{E}\left(\widetilde{b}_{H}, \widetilde{c}_{H}\right)$ 
Proof. The proof is obvious.

3.2. Description of MADM Problem. Consider a decisionmaking problem consisting of $m$ number of alternatives $\mathscr{A}_{1}, \mathscr{A}_{2}, \ldots, \mathscr{A}_{m}$ and are assessed under the $n$ different attributes $\mathscr{B}_{1}, \mathscr{B}_{2}, \ldots, \mathscr{B}_{n}$. The weight information of these $n$ attributes are represented in terms of weight vector $w_{j}>0$ with $\sum_{j=1}^{n} w_{j}=1$. The given attributes are categorized into two parts, namely, the benefit (B) and the cost (C) attributes, such that $B \cap C=\varnothing$. The given alternative $\mathscr{A}_{i}$ is evaluated by an expert under each attribute $\mathscr{B}_{j}$ and provides their relative information in terms of the hesitant fuzzy information denoted by $d_{i j}$. Mathematically, the obtained information from an expert corresponding to $i^{\text {th }}$ alternative is represented as

$$
d_{i}=\left\{\left(\mathscr{B}_{1}, d_{11}\right),\left(\mathscr{B}_{2}, d_{12}\right), \ldots,\left(\mathscr{B}_{n}, d_{1 n}\right)\right\} ; \quad i=1,2, \ldots, m .
$$

The overall collective information of each alternative under different attribute is summarized in the decision matrix $D=\left(d_{\mathrm{ij}}\right)_{m \times n}$ as

$$
D=\left(\begin{array}{cccc}
d_{11} & d_{12} & \ldots & d_{1 n} \\
d_{21} & d_{22} & \ldots & d_{2 n} \\
\vdots & \vdots & \ddots & \vdots \\
d_{m 1} & d_{m 2} & \ldots & d_{\mathrm{mn}}
\end{array}\right) .
$$

The target of the problem is to determine the best alternative(s) among the feasible ones. There are several methods exist in the literature to solve MADM problems. In Section 3.3, we stated the TOPSIS, one of such methods, to solve the MADM problems using the concept of the relative closeness degrees.

3.3. Standard TOPSIS Method. The TOPSIS method is one of the widely used methods to solve the MADM problem. In this method, the relative strength of each alternative is computed from its positive ideal solution (PIS) and the negative ideal solution (NIS) by using the distance measures. Then, based on their measures values, a relative closeness degree is evaluated for each alternative to rank the given numbers. In the brief, the basic steps associated with the standard TOPSIS approach [44] is discussed as follows:

(i) Step 1: summarize the information in terms of decision matrix $D=\left(d_{\mathrm{ij}}\right)_{m \times n}$ as

$$
D=\left(\begin{array}{cccc}
d_{11} & d_{12} & \ldots & d_{1 n} \\
d_{21} & d_{22} & \ldots & d_{2 n} \\
\vdots & \vdots & \ddots & \vdots \\
d_{m 1} & d_{m 2} & \ldots & d_{\mathrm{mn}}
\end{array}\right) .
$$

(ii) Step 2: normalized the matrix, if needed, to $N=\left(n_{\mathrm{ij}}\right)_{m \times n}$, where

$$
n_{i j}= \begin{cases}\frac{d_{j}^{\max }-d_{i j}}{d_{j}^{\max }-d_{j}^{\min } ;} & j \in B, \\ \frac{d_{i j}-d_{j}^{\min }}{d_{j}^{\max }-d_{j}^{\min } ;} & j \in C,\end{cases}
$$

where $d_{j}^{\max }=\max _{i}\left\{d_{\mathrm{ij}}\right\}$.

(iii) Step 3: calculate the weighted normalized decision matrix NW $=\left(v_{i j}\right)_{m \times n}$, where $v_{i j}$ is computed as

$$
v_{i j}=n_{i j} \times w_{i} .
$$

(iv) Step 4: compute the two subjective alternatives PIS $A^{+}$and NIS $A^{-}$as

$$
\begin{aligned}
& \mathscr{A}^{+}=\left\{d_{1}^{+}, d_{2}^{+}, \ldots, d_{m}^{+}\right\}=\left\{\left(\max _{i} v_{i j} \mid j \in B\right) \&\left(\min _{i} v_{i j} \mid j \in C\right)\right\}, \\
& \mathscr{A}^{-}=\left\{d_{1}^{-}, d_{2}^{-}, \ldots, d_{m}^{-}\right\}=\left\{\left(\min _{i} v_{i j} \mid j \in B\right) \&\left(\max _{i} v_{i j} \mid j \in C\right)\right\} .
\end{aligned}
$$

(v) Step 5: calculate the Euclidean distance of each alternative from subjective alternatives, where

$$
\begin{aligned}
& S_{i}^{+}=\sqrt{\sum_{j=1}^{m}\left(v_{i j}-d_{j}^{+}\right)^{2}}, \\
& S_{i}^{-}=\sqrt{\sum_{j=1}^{m}\left(v_{i j}-d_{j}^{-}\right)^{2}}, \quad i=1,2, \ldots, m .
\end{aligned}
$$

(vi) Step 6: calculate the closeness degree $r_{i}=\left(S_{i}^{-} / S_{i}^{+}+S_{i}^{-}\right), \quad i=1,2, \ldots, m$ and rank them in the ascending order.

3.4. Measure of Choquet Integral. In some practical problems, the criteria have an effect on each other. In other words, the weight of the coalition is not equal to the total weight of its members. To address such kinds of problem, a Choquet integral (CI) method, using nonadditivity property of fuzzy measure, has been proposed [44].

Definition 11 (see [47]). Let $X$ be a set. For any $H, J \in P(X)$. A map $\mu: P(X) \longrightarrow[0,1]$ where

$$
\begin{aligned}
& \varnothing \in P(X) \Longrightarrow \mu(\varnothing)=0, \\
& \text { If } P(X) \supseteq H \supseteq J, \quad \text { then } \mu(H) \geq \mu(J),
\end{aligned}
$$

is called a fuzzy measure.

Definition 12 (see [47]). Let $\mu$ be a fuzzy measure, and for any $H, J \in P(X)$, we can define

$$
\mu(H \cup J)=\mu(H)+\mu(J)+\lambda \mu(H \cap J),
$$


where $\lambda$ represents the interaction between the parameters. The basic features of $\mu$ are

(1) Additive: if $H \cap J=\varnothing$, then $\mu(H \cup J)=\mu(H)+\mu(J)$

(2) Subadditive: if $\quad H \cap J=\varnothing$, then $\mu(H \cup J) \leq \mu(H)+\mu(J)$

(3) Supermodular: $\mu(H \cup J)+\mu(H \cap J) \geq \mu(H)+\mu(J)$

(4) Submodular: $\mu(H \cup J)+\mu(H \cap J) \leq \mu(H)+\mu(J)$

A $\lambda$-fuzzy measure, in which $\lambda$ must be obtained from solving the equation $1+\lambda=\Pi_{i=1}^{n}\left(1+\lambda \mu\left\{x_{i}\right\}\right)=$ $\Pi_{i=1}^{n}\left(1+\lambda w_{i}\right)$, enables us to compute qualition weights $\mu(E \cup F)$ of $E, F \subset P(X)$, based on the given weights $\mu(E), \mu(F)$, as $\mu(E \cup F)=\mu(E)+\mu(F)+\lambda \mu(E) \mu(F)$.

The Choquet integral (CI) is a subadditive and superadditive integral which is an adequate tool to measure the mean of a set and hence to assess the features of an object scientifically. Under CI, each item is ordered in the descending order, and weights are regulated by the measure $\mu$. Related to $\mu$, the CI is described as follows.

Definition 13 (see [48]). Let $f$ be the defined function on $X$ with values $f\left(x_{1}\right), f\left(x_{2}\right), \ldots, f\left(x_{n}\right)$ which are reordered as $f\left(x_{1}^{*}\right) \leq f\left(x_{2}^{*}\right) \leq \cdots \leq f\left(x_{n}^{*}\right)$, and $\left\{x_{1}^{*}, x_{2}^{*}, \ldots, x_{n}^{*}\right\}$ is a permutation of $\left\{x_{1}, x_{2}, \ldots, x_{n}\right\}$ such that the above conditions be true, then

$$
\text { (C) } \int f d \mu=\sum_{i=1}^{n} f\left[\left(x_{i}^{*}\right)-f\left(x_{i-1}^{*}\right)\right] \cdot \mu\left(\left\{x_{i}^{*}, x_{i+1}^{*}, \ldots, x_{n}^{*}\right\}\right),
$$

is called the CI of $f$ on $X$.

This is a powerful tool not only in aggregation of assessment values of interactive attribute, but also under certain conditions, it becomes to other aggregation functions. For instance, $\mathrm{CI}$ is called $\min (\max )$ operator, if for each $A \subseteq X: \mu(A)=0, \mu(X)=1 \quad(\mu(\varnothing)=0$ and for each $A \subseteq X: \mu(A)=1)$, if we define for each $x_{i} \in X, \mu\left(x_{i}\right)=w_{i}$ with $\sum_{i} w_{i}=1$, and then $\mathrm{CI}$ is the weighted averaging (WA) operator, i.e. $\mu$ has an additivity property. When fuzzy measure $\mu(A)$ is related to cardinal number of set $A$, that is, the sets with the same number of elements have the same measure, then $w_{i}=\mu\left(A_{i}\right)-\mu\left(A_{i-1}\right)$, where $A_{i}$ is the subset of $X$ with $\operatorname{Card}\left(A_{i}\right)=i$, and then CI converts to ordered weighted averaging (OWA) [49].

3.5. Proposed MADM Algorithm. In this section, we have addressed a new MADM algorithm by combining the TOPSIS and CI methods together called as TOPSIS-CI method, to solve an MADM problem. In the presented approach, the interaction between the different attributes is considered to be interactive with each other.

Consider a MADM problem described in Section 3.2 with $m$ alternatives $\mathscr{A}_{i}$ and $n$ attributes $\mathscr{B}_{j}$ where $i=1,2, \ldots, m$ and $j=1,2, \ldots, n$. Let the evaluation value of $i$ th alternative against $j$ th criterion is modeled by HFN $\widetilde{h}_{\mathrm{ij}}=$ $\left\langle a_{\mathrm{ij}}, h\left(a_{\mathrm{ij}}\right)\right\rangle$ which are arranged in a matrix called decision matrix, $H=\left(\widetilde{h}_{\mathrm{ij}}\right)_{m \times n}$ as

$$
D=\left(\begin{array}{cccc}
h_{11} & h_{12} & \ldots & h_{1 n} \\
h_{21} & h_{22} & \ldots & h_{2 n} \\
\vdots & \vdots & \ddots & \vdots \\
h_{m 1} & h_{m 2} & \ldots & h_{m n}
\end{array}\right) .
$$

The procedure steps of the proposed TOPSIS-CI method to access the best alternative(s) are summarized as follows:

(i) Step 1 (normalization): for the benefit (B) and the cost $(\mathrm{C})$ attributes, we normalized the given ratings by using equation (23) and obtained the normalized decision matrix $\mathrm{NH}=\left(\widetilde{n}_{\mathrm{ij}}\right)_{m \times n}$ :

$$
\left(\tilde{n h}_{i j}\right)=\left\langle n a_{i j}, h\left(a_{i j}\right)\right\rangle= \begin{cases}\left\langle\frac{a_{j}^{\max }-a_{i j}}{a_{j}^{\max }-a_{j}^{\min }}, h\left(a_{i j}\right)\right\rangle ; & j \in B, \\ \left\langle\frac{a_{i j}-a_{j}^{\min }}{a_{j}^{\max }-a_{j}^{\min }}, h\left(a_{i j}\right)\right\rangle ; & j \in C,\end{cases}
$$

where $a_{j}^{\min }=\min _{i}\left\{a_{i j}\right\}$ and $a_{j}^{\max }=\max _{i}\left\{a_{i j}\right\}$.

(ii) Step 2 (ideal alternative): determine the PIS $\mathscr{A}^{+}$and the NIS $\mathscr{A}^{-}$as two subjective alternatives:

$$
\begin{aligned}
\mathscr{A}^{+} & =\left\{\tilde{d}_{1}^{+}, \tilde{d}_{2}^{+}, \ldots, \tilde{d}_{m}^{+}\right\}, \\
& \left\{\begin{array}{c}
\left\langle\max _{i}\left\{n a_{i j}\right\},\left\{\gamma \mid \gamma \in \cup_{i} h\left(a_{i j}\right) \backslash \gamma \geq 0.5\right\} \mid j \in B\right\rangle \\
\left\langle\min _{i}\left\{n a_{i j}\right\},\left\{\gamma \mid \gamma \in \cup_{i} h\left(a_{i j}\right) \backslash \gamma<0.5\right\} \mid j \in C\right\rangle
\end{array}\right\}, \\
\mathscr{A}^{-} & =\left\{\tilde{d}_{1}^{-}, \tilde{d}_{2}^{-}, \ldots, \tilde{d}_{m}^{-}\right\}, \\
& \left\{\begin{array}{l}
\left\langle\max _{i}\left\{n a_{i j}\right\},\left\{\gamma \mid \gamma \in \cup_{i} h\left(a_{i j}\right) \backslash \gamma \geq 0.5\right\} \mid j \in B\right\rangle \\
\left\langle\min _{i}\left\{n a_{i j}\right\},\left\{\gamma \mid \gamma \in \cup_{i} h\left(a_{i j}\right) \backslash \gamma<0.5\right\} \mid j \in C\right\rangle
\end{array}\right\} .
\end{aligned}
$$

(iii) Step 3 (distance metrics): construct positive distance matrix $\mathrm{PD}^{+}=\left(d_{i j}^{+}\right)_{m \times n}$ and negative distance matrix $\mathrm{PD}^{-}=\left(d_{i j}^{-}\right)_{m \times n}$ from the PIS and the NIS as follows:

$$
\begin{aligned}
\mathrm{PD}^{+} & =\left(\begin{array}{cccc}
d_{11}^{+} & d_{12}^{+} & \ldots & d_{1 n}^{+} \\
d_{21}^{+} & d_{22}^{+} & \ldots & d_{2 n}^{+} \\
\vdots & \vdots & \ddots & \vdots \\
d_{m 1}^{+} & d_{m 2}^{+} & \ldots & d_{\mathrm{mn}}^{+}
\end{array}\right), \\
\mathrm{PD}^{-} & =\left(\begin{array}{cccc}
d_{11}^{-} & d_{12}^{-} & \ldots & d_{1 n}^{-} \\
d_{21}^{-} & d_{22}^{-} & \ldots & d_{2 n}^{-} \\
\vdots & \vdots & \ddots & \vdots \\
d_{m 1}^{-} & d_{m 2}^{-} & \ldots & d_{\mathrm{mn}}^{-}
\end{array}\right),
\end{aligned}
$$

where $d_{i j}^{+}$is the distance between $d_{i j}$ and $d_{j}^{+}$and $d_{i j}^{-}$ is the distance between $d_{i j}$ and $d_{j}^{-}$, for $j=1,2, \ldots, n$ by using the proposed measures given in equations 
(10) and (11) as $d_{i j}^{+}=d_{E}\left(\tilde{n h}_{i j}, \tilde{d}_{j}^{+}\right)\left(\right.$or $d_{i j}^{+}=d_{H}\left(\tilde{n h}_{i j}\right.$, $\left.\left.\widetilde{d}_{j}^{+}\right)\right)$and $d_{i j}^{-}=d_{E}\left(n h_{i j}, \widetilde{d}_{j}^{-}\right)\left(\right.$or $\left.d_{i j}^{-}=d_{H}\left(\tilde{n h}_{i j}, \widetilde{d}_{j}^{-}\right)\right)$.

(iv) Step 4 (positive and negative distances): utilizing the weight vector $W$ of the attributes, aggregate each row of the distance matrices $\mathrm{PD}^{+}$and $\mathrm{PD}^{-}$using $\mathrm{CI}$ method as

$$
\begin{aligned}
& S_{i}^{+}=(C) \int d_{i}^{+} d \mu=\sum_{j=1}^{n}\left[\mu\left[\mathscr{B}_{(j)}\right]-\mu\left(\mathscr{B}_{(j+1)}\right)\right] d_{i(j)}^{+}, \\
& S_{i}^{-}=(C) \int d_{i}^{-} d \mu=\sum_{j=1}^{n}\left[\mu\left(\mathscr{B}_{(j)}\right)-\mu\left(\mathscr{B}_{(j+1)}\right)\right] d_{i(j)}^{-},
\end{aligned}
$$

where $d_{i(1)}^{+}, d_{i(2)}^{+}, \ldots, d_{i(n)}^{+}$are permutation of $d_{i 1}^{+}, d_{i 2}^{+}, \ldots, d_{i n}^{+}$such that $d_{i(1)}^{+} \leq d_{i(2)}^{+} \leq \ldots \leq d_{i(n)}^{+}$, $d_{i(1)}^{-}, d_{i(2)}^{-}, \ldots, d_{i(n)}^{-}$are permutation of $d_{i 1}^{-}, d_{i 2}^{-}, \ldots, d_{\text {in }}^{-}$such that $d_{i(1)}^{-} \leq d_{i(2)}^{-} \leq \cdots \leq d_{i(n)}^{-}$, and $\mu\left(\mathscr{B}_{(j)}\right)$ is the joint measure (weight) of attribute $\mathscr{B}_{(j)}, \mathscr{B}_{(j+1)}, \ldots, \mathscr{B}_{(n)}$ that are obtained from weight vector $W$ using $\lambda$-fuzzy measure.

(v) Step 5 (relative closeness): compute the relative distance of $i$ th alternative $(i=1,2, \ldots, m)$ from subjective alternatives PIS and NIS as

$$
r_{i}=\left(\frac{S_{i}^{-}}{S_{i}^{+}+S_{i}^{-}}\right), \quad i=1,2, \ldots, n,
$$

provided $S_{i}^{+} \neq 0$.

(vi) Step 6 (ranking): rank the alternatives based on ascending order values of $r_{i}, i=1,2, \ldots, m$.

\section{Numerical Example}

In this section, the abovementioned TOPSIS-CI approach has been illustrated with a numerical example as follows.

4.1. A Case Study. Suppose that there are seven organizations (alternatives) $\mathscr{A}_{i}(i=1,2, \ldots, 7)$ and six attributes: $\mathscr{B}_{1}$ (improving organizational structure), $\mathscr{B}_{2}$ (development of e-government), $\mathscr{B}_{3}$ (human capital management), $\mathscr{B}_{4}$ (improving business environment and productivity), $\mathscr{B}_{5}$ (improving administrative health, responsibility, and accountability), and $\mathscr{B}_{6}$ (establishment of a performance management system). Let $W=(0.125,0.33,0.175,0.09,0.15$, and 0.13$)$ be the weight vector of attributes. It is assumed that the alternative $\mathscr{A}_{5}$ is a virtual option with unrealistic self-assessment scores. To evaluate the performance of organizations, predesigned forms are provided to organizations based on the mentioned attribute. The score ceiling for each of the attribute in the designed forms, which also have subattribute, is $125,330,175,90,150$, and 130 , respectively. Organizations evaluate themselves according to the available forms and upload the relevant documents. The obtained values are summed up in a decision matrix $D=\left(h_{i j}\right)_{7 \times 6}$ :

$$
\begin{aligned}
& \begin{array}{cccccc}
\mathcal{B}_{1} & \mathcal{B}_{2} & \mathcal{B}_{3} & \mathcal{B}_{4} & \mathcal{B}_{5} & \mathcal{B}_{6}
\end{array} \\
& \mathcal{A}_{1}\left(\begin{array}{llllll}
124 & 321 & 175 & 82 & 144 & 127
\end{array}\right) \\
& \begin{array}{lllllll}
\mathcal{A}_{2} & 123 & 324 & 160 & 88 & 147 & 125
\end{array} \\
& \begin{array}{l|llllll}
\mathcal{A}_{3} & 123 & 327 & 170 & 85 & 148 & 127
\end{array} \\
& \begin{array}{l|llllll}
D=\mathcal{A}_{4} & 121 & 329 & 173 & 85 & 147 & 129
\end{array} \\
& \begin{array}{l|llllll}
\mathcal{A}_{5} & 125 & 327 & 171 & 90 & 149 & 130
\end{array} \\
& \begin{array}{lllllll}
\mathcal{A}_{6} & 125 & 325 & 172 & 90 & 145 & 129
\end{array} \\
& \mathcal{A}_{7}\left(\begin{array}{llllll}
122 & 325 & 172 & 89 & 147 & 129
\end{array}\right)
\end{aligned}
$$

All the elements of decision matrix $D$ are merely assessment values, so we do not need to normalize them. Further, given that each organization has enough motivation to show reality in order to earn higher scores, in this paper, we invited five experts to evaluate the concessions allocated from the perspective of compliance with the existing reality and expressed their opinions in this regard with values of the range of 0 and 1 . Thus, decision matrix $D=\left(d_{i j}\right)_{7 \times 6}$ with real elements becomes to hesitant decision matrix $\widehat{\mathrm{HD}}=\left(\left\langle d_{i j},\left\{\gamma_{1}, \ldots, \gamma_{5}\right\}\right\rangle\right)_{7 \times 6}$, with HFN elements in which $\gamma_{i}$ is the opinion of $i$ th expert as follows: 


$$
\begin{aligned}
\widetilde{\mathrm{HD}}= & \left\langle\begin{array}{llll}
\langle 124 ;\{0.3,0.4,0.5,0.5,0.2\}\rangle & \langle 321 ;\{0.1,0.4,0.7,0.8,0.9\}\rangle & \langle 175 ;\{0.2,0.6,0.6,0.4,0.5\}\rangle \\
\langle 123 ;\{0.3,0.5,0.8,0.6,0.9\}\rangle & \langle 324 ;\{0.3,0.5,0.6,0.5,0.9\}\rangle & \langle 160 ;\{0.9,0.9,0.9,0.9,0.9\}\rangle \\
\langle 123 ;\{0.3,0.5,0.6,0.7,0.9\}\rangle & \langle 327 ;\{0.1,0.5,0.6,0.9,0.9\}\rangle & \langle 170 ;\{0.3,0.5,0.7,0.6,0.9\}\rangle \\
\langle 121 ;\{0.9,0.7,0.8,0.9,0.9\}\rangle & \langle 329 ;\{0.1,0.7,0.3,0.8,0.9\}\rangle & \langle 173 ;\{0.2,0.6,0.7,0.4,0.5\}\rangle \\
\langle 125 ;\{0.1,0.1,0.1,0.1,0.1\}\rangle & \langle 327 ;\{0.1,0.2,0.1,0.2,0.1\}\rangle & \langle 171 ;\{0.2,0.2,0.3,0.1,0.1\}\rangle \\
\langle 125 ;\{0.8,0.8,0.9,0.8,0.9\}\rangle & \langle 325 ;\{0.9,0.8,0.7,0.8,0.9\}\rangle & \langle 172 ;\{0.2,0.2,0.3,0.4,0.5\}\rangle \\
\langle 122 ;\{0.4,0.4,0.5,0.6,0.9\}\rangle & \langle 325 ;\{0.8,0.5,0.6,0.9,0.6\}\rangle & \langle 172 ;\{0.3,0.5,0.6,0.6,0.6\}\rangle \\
& \langle 82 ;\{0.7,0.7,0.5,0.6,0.9\}\rangle & \langle 144 ;\{0.3,0.2,0.6,0.3,0.3\}\rangle & \langle 127 ;\{0.3,0.4,0.6,0.7,0.7\}\rangle \\
& \langle 88 ;\{0.7,0.8,0.5,0.5,0.9\}\rangle & \langle 147 ;\{0.3,0.4,0.4,0.6,0.8\}\rangle & \langle 125 ;\{0.8,0.8,0.8,0.9,0.9\}\rangle \\
& \langle 85 ;\{0.7,0.6,0.5,0.6,0.9\}\rangle & \langle 148 ;\{0.3,0.3,0.5,0.6,0.6\}\rangle & \langle 127 ;\{0.6,0.7,0.8,0.6,0.9\}\rangle \\
& \langle 85 ;\{0.8,0.7,0.5,0.5,0.6\}\rangle & \langle 147 ;\{0.1,0.5,0.4,0.5,0.9\}\rangle & \langle 129 ;\{0.7,0.3,0.3,0.4,0.5\}\rangle \\
& \langle 90 ;\{0.1,0.2,0.2,0.2,0.2\}\rangle & \langle 149 ;\{0.1,0.2,0.1,0.3,0.3\}\rangle & \langle 130 ;\{0.3,0.1,0.2,0.2,0.1\}\rangle \\
& \langle 90 ;\{0.3,0.4,0.5,0.5,0.2\}\rangle & \langle 145 ;\{0.1,0.7,0.6,0.8,0.9\}\rangle & \langle 129 ;\{0.5,0.3,0.7,0.4,0.5\}\rangle \\
& \langle 89 ;\{0.3,0.5,0.5,0.6,0.9\}\rangle & \langle 147 ;\{0.7,0.7,0.5,0.6,0.8\}\rangle & \langle 129 ;\{0.3,0.5,0.6,0.4,0.4\}\rangle
\end{array}\right) .
\end{aligned}
$$

Then, the steps of the proposed algorithm are implemented here to find the best organization: (ii) Step 2: compute the PIS $\mathscr{A}^{+}$and NIS $\mathscr{A}^{-}$alternatives by using equations (24) and (25) and get

(i) Step 1: because data are scaleless, normalization is not necessary

$$
\begin{aligned}
& \mathscr{A}^{+}=\left\{\begin{array}{c}
\langle 125 ;\{1.0,1.0,1.0,1.0,1.0\}\rangle,\langle 330 ;\{1.0,1.0,1.0,1.0,1.0\}\rangle,\langle 175 ;\{1.0,1.0,1.0,1.0,1.0\}\rangle \\
\langle 90 ;\{1.0,1.0,1.0,1.0,1.0\}\rangle,\langle 150 ;\{1.0,1.0,1.0,1.0,1.0\}\rangle,\langle 130 ;\{1.0,1.0,1.0,1.0,1.0\}\rangle
\end{array}\right\}, \\
& \mathscr{A}^{-}=\left\{\begin{array}{c}
\langle 121 ;\{0.1,0.1,0.1,0.1,0.1\}\rangle,\langle 321 ;\{0.1,0.2,0.1,0.2,0.1\}\}\rangle,\langle 160 ;\{0.2,0.2,0.3,0.1,0.1\}\rangle \\
\langle 82 ;\{0.1,0.2,0.2,0.2,0.2\}\rangle,\langle 144 ;\{0.1,0.2,0.1,0.3,0.3\}\rangle,\langle 125 ;\{0.3,0.1,0.2,0.2,0.1\}\rangle
\end{array}\right\} .
\end{aligned}
$$

(iii) Step 3: by utilizing the hamming distance measure as defined in equation (10), the $\mathrm{PD}^{+}$and $\mathrm{PD}^{-}$ metrics are computed as

$$
\begin{aligned}
& \begin{array}{llllll}
\mathcal{B}_{1} & \mathcal{B}_{2} & \mathcal{B}_{3} & \mathcal{B}_{4} & \mathcal{B}_{5} & \mathcal{B}_{6}
\end{array}
\end{aligned}
$$

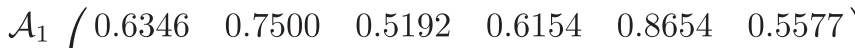

$$
\begin{aligned}
& \begin{array}{lllllll}
\mathcal{A}_{2} & 0.4423 & 0.6538 & 0.6731 & 0.3846 & 0.5962 & 0.3462
\end{array} \\
& \begin{array}{llllllll}
\mathcal{A}_{3} & 0.4615 & 0.4615 & 0.5769 & 0.5192 & 0.5962 & 0.3846
\end{array} \\
& P D^{+}=\mathcal{A}_{4} \quad \begin{array}{lllllll}
0.3095 & 0.4615 & 0.5769 & 0.5577 & 0.6154 & 0.5769
\end{array} \\
& \begin{array}{l|llllll}
\mathcal{A}_{5} & 0.3269 & 0.4808 & 0.5769 & 0.4615 & 0.4423 & 0.6731
\end{array} \\
& \begin{array}{l|llllll}
\mathcal{A}_{6} & 0.1538 & 0.3654 & 0.7692 & 0.5962 & 0.5577 & 0.5385
\end{array} \\
& \mathcal{A}_{7}\left(\begin{array}{llllll}
0.5385 & 0.5000 & 0.5769 & 0.4615 & 0.4423 & 0.5769
\end{array}\right) \\
& \begin{array}{cccccc}
\mathcal{B}_{1} & \mathcal{B}_{2} & \mathcal{B}_{3} & \mathcal{B}_{4} & \mathcal{B}_{5} & \mathcal{B}_{6}
\end{array} \\
& \left.\begin{array}{rl|llllll}
\mathcal{A}_{1} & 0.3846 & 0.4385 & 0.8538 & 0.4808 & 0.1500 & 0.4231 \\
\mathcal{A}_{2} & 0.5769 & 0.5192 & 0.6923 & 0.7115 & 0.4038 & 0.6346 \\
\mathcal{A}_{3} & 0.5577 & 0.6885 & 0.7885 & 0.5769 & 0.4038 & 0.5962 \\
\mathcal{P}^{-}= & \mathcal{A}_{4} & 0.8269 & 0.7269 & 0.7962 & 0.5385 & 0.4231 & 0.4038 \\
\mathcal{A}_{5} & 0.1538 & 0.2769 & 0.5000 & 0.3385 & 0.2846 & 0.2692 \\
\mathcal{A}_{6} & 0.8654 & 0.8077 & 0.6115 & 0.5000 & 0.4808 & 0.4423 \\
& \mathcal{A}_{7} & 0.4808 & 0.6731 & 0.7885 & 0.6346 & 0.5577 & 0.4038
\end{array}\right)
\end{aligned}
$$

(31) 
(iv) Step 4: by using equations (27) and (28) and the values of $\mathrm{PD}^{+}$and $\mathrm{PD}^{-}$, we get the measurement values as

$$
\begin{aligned}
& S_{1}^{+}=0.6754, \\
& S_{2}^{+}=0.5579, \\
& S_{3}^{+}=0.4971, \\
& S_{4}^{+}=0.5094, \\
& S_{5}^{+}=0.4959, \\
& S_{6}^{+}=0.4817, \\
& S_{7}^{+}=0.5161, \\
& S_{1}^{-}=0.4630, \\
& S_{2}^{-}=0.4299, \\
& S_{3}^{-}=0.6249, \\
& S_{4}^{-}=0.6470, \\
& S_{5}^{-}=0.3063, \\
& S_{6}^{-}=0.6563, \\
& S_{7}^{-}=0.6135,
\end{aligned}
$$

(v) Step 5: by equation (29), we get

$$
\begin{aligned}
& r_{1}=0.4067, \\
& r_{2}=0.4352, \\
& r_{3}=0.5569, \\
& r_{4}=0.5595, \\
& r_{5}=0.3818, \\
& r_{6}=0.5767, \\
& r_{7}=0.5431 .
\end{aligned}
$$

(vi) Step 6: since $r_{6}>r_{4}>r_{3}>r_{7}>r_{2}>r_{1}>r_{5}$ and thus the ranking order of the given alternative (organizations) is $\mathscr{A}_{6}>\mathscr{A}_{4}>\mathscr{A}_{3}>\mathscr{A}_{7}>\mathscr{A}_{2}>\mathscr{A}_{1}>\mathscr{A}_{5}$, the best alternative is $\mathscr{A}_{6}$.

4.2. Validity Test. Checking and demonstrating the feasibility of MCDM methods will be accomplished through the following three methods proposed by Wang and Triantaphyllou [5].

(i) Test criterion 1: the rank of the best option should be unchanged, if the weight vector do not change, and a nonoptimal option was replaced with the worse one.

(ii) Test criterion 2: the transitivity property should be true in an effective MCDM method.

(iii) Test criterion 3: an effective MCDM method gives similar rankings in each of the following two cases: (i) solving the given MCDM problem without breaking it down into several MCDM subproblems; (ii) solving the resulting MCDM subproblems from decomposition of original MCDM problem and combining their rankings.

Let $\mathscr{A}$ with the following self-assessment' points vector defined as

$$
\mathscr{A}=\left\{\begin{array}{c}
\langle 122 ;\{0.4,0.7,0.2,0.5,0.5\}\rangle,\langle 324 ;\{0.5,0.2,0.3,0.6,0.3\}\rangle,\langle 169 ;\{0.3,0.8,0.6,0.6,0.5\}\rangle, \\
\langle 87 ;\{0.4,0.9,0.1,0.2,0.2\}\rangle,\langle 145 ;\{0.6,0.6,0.5,0.3,0.3\}\rangle,\langle 126 ;\{0.4,0.7,0.6,0.7,0.7\}\rangle
\end{array}\right\},
$$

and is a nonoptimal organization which has replaced $\mathscr{A}_{3}$. Now, based on the updated information, if we apply the steps of the proposed TOPSIS-CI method, then we get its positive and negative distance vectors from PIS and NIS $d_{\mathscr{A}}^{+}=(0.6346,0.8269,0.6538,0.7308,0.7115,0.5192)$ and $d_{\mathscr{d}}^{-}=(0.3846,0.3462,0.7115,0.3962,0.2885,0.4615)$ for the given alternative. Based on it, we compute $S_{\mathscr{A}}^{+}=0.7060$ and $S_{\mathscr{A}}^{-}=0.4253$, and hence the closeness degree $r_{\mathscr{A}}=0.3759$. Based on the above calculated closeness degrees, we found that, in the ranking order of the alternative with this new $\mathscr{A}$, we get that the position of the best organization is unchanged.
Further, if we decomposed the problem into subproblems $\quad\left\{\mathscr{A}_{1}, \mathscr{A}_{2}, \mathscr{A}_{5}, \mathscr{A}_{6}, \mathscr{A}_{7}\right\}, \quad\left\{\mathscr{A}_{3}, \mathscr{A}_{4}, \mathscr{A}_{5}, \mathscr{A}_{7}\right\}$, $\left\{\mathscr{A}_{1}, \mathscr{A}_{2}, \mathscr{A}_{3}, \mathscr{A}_{4}, \mathscr{A}_{5}, \mathscr{A}_{7}\right\}$, and $\left\{\mathscr{A}_{2}, \mathscr{A}_{3}, \mathscr{A}_{4}, \mathscr{A}_{5}, \mathscr{A}_{7}\right\}$. By implementing the steps of the proposed TOPSIS-CI method, the final ranking order of these subproblems are obtained as $\mathscr{A}_{6}>\mathscr{A}_{7}>\mathscr{A}_{2}>\mathscr{A}_{1}>\mathscr{A}_{5}, \quad \quad \mathscr{A}_{7}>\mathscr{A}_{4}>\mathscr{A}_{3}>\mathscr{A}_{5}$, $\mathscr{A}_{4}>\mathscr{A}_{3}>\mathscr{A}_{7}>\mathscr{A}_{2}>\mathscr{A}_{1}>\mathscr{A}_{5}$, and $\mathscr{A}_{7}>\mathscr{A}_{4}>\mathscr{A}_{3}>\mathscr{A}_{2}>\mathscr{A}_{5}$, respectively. Hence, the overall ranking order of organizations will be achieved through the combining these orders as $\mathscr{A}_{6}>\mathscr{A}_{4}>\mathscr{A}_{3}>\mathscr{A}_{7}>\mathscr{A}_{2}>\mathscr{A}_{1}>\mathscr{A}_{5}$ which is the same as the original ranking order. Therefore, the proposed method is valid under test criteria 1,2 , and 3 . 
4.3. Numerical Analysis and Comparative Study. In this section, the problem will be solved from two separate angles: only from the point of view of self-assessment scores, i.e., decision matrix $D$ will be used and other from the point of view of decision makers, i.e., decision matrix $\widetilde{H}$ will be used.

In the former case, direct sum $\sum_{i} d_{i j}$ of $i$ th row of matrix $D$ is called the score of $i$ th alternative which is used to rank the given alternatives. For it, we computed the score from the matrix $D$ and get their results as score $\left(\mathscr{A}_{1}\right)=973$, score $\left(\mathscr{A}_{2}\right)=967$, score $\left(\mathscr{A}_{3}\right)=980$, score $\left(\mathscr{A}_{4}\right)=984$, score $\left(\mathscr{A}_{5}\right)=992$, score $\left(\mathscr{A}_{6}\right)=986$, and score $\left(\mathscr{A}_{7}\right)=984$. Thus, based on these computed score values, we concluded that the ranking order of the alternative is $\mathscr{A}_{5}>\mathscr{A}_{6}>\mathscr{A}_{7}=\mathscr{A}_{4}>\mathscr{A}_{3}>\mathscr{A}_{1}>\mathscr{A}_{2}$.

Secondly, we utilized the simple additive weighting method to rank the given numbers. For it, by using the weight vector $w_{j}>0$, the rating information of the $i$ th row of matrix $D$ is aggregated by using $\sum_{j} w_{j} d_{i j}$. Hence, the order of the alternative is obtained from their aggregated values and $\mathscr{A}_{5}>\mathscr{A}_{4}>\mathscr{A}_{6}>\mathscr{A}_{7}>\mathscr{A}_{3}>\mathscr{A}_{1}>\mathscr{A}_{2}$ is obtained.

However, if we apply the proposed TOPSIS-CI method on the matrix $D$ with weight vector $W$, then the final ranking order of the alternatives is obtained as $\mathscr{A}_{5}>\mathscr{A}_{3}>\mathscr{A}_{4}>\mathscr{A}_{6}>\mathscr{A}_{7}>\mathscr{A}_{1}>\mathscr{A}_{2}$.

From these three methods, direct sum, simple additive weighting, and proposed TOPSIS-CI, we conclude from their final ranking order that the virtual option $\mathscr{A}_{5}$ with unrealistic points is the best alternative. This means that unrealistic scores and individual motivations can affect the final ranking, encouraging other organizations to document incorrectly.

In the latter case, if we analyze the alternative behavior by considering only the opinions of decision makers, then for it firstly the given decision matrix has been converted to hesitant decision matrix $\widetilde{H}$, by omitting the real component of the HFNs, and the matrix $\tilde{H}$ is obtained as follows:

$$
\begin{aligned}
\widetilde{H}= & \left(\begin{array}{llll}
\langle\{0.3,0.4,0.5,0.5,0.2\}\rangle & \langle\{0.1,0.4,0.7,0.8,0.9\}\rangle & \langle\{0.2,0.6,0.6,0.4,0.5\}\rangle \\
\langle\{0.3,0.5,0.8,0.6,0.9\}\rangle & \langle\{0.3,0.5,0.6,0.5,0.9\}\rangle & \langle\{0.9,0.9,0.9,0.9,0.9\}\rangle \\
\langle\{0.3,0.5,0.6,0.7,0.9\}\rangle & \langle\{0.1,0.5,0.6,0.9,0.9\}\rangle & \langle\{0.3,0.5,0.7,0.6,0.9\}\rangle \\
\langle\{0.9,0.7,0.8,0.9,0.9\}\rangle & \langle\{0.1,0.7,0.3,0.8,0.9\}\rangle & \langle\{0.2,0.6,0.7,0.4,0.5\}\rangle \\
\langle\{0.1,0.1,0.1,0.1,0.1\}\rangle & \langle\{0.1,0.2,0.1,0.2,0.1\}\rangle & \langle\{0.2,0.2,0.3,0.1,0.1\}\rangle \\
\langle\{0.8,0.8,0.9,0.8,0.9\}\rangle & \langle\{0.9,0.8,0.7,0.8,0.9\}\rangle & \langle\{0.2,0.2,0.3,0.4,0.5\}\rangle \\
\langle\{0.4,0.4,0.5,0.6,0.9\}\rangle & \langle\{0.8,0.5,0.6,0.9,0.6\}\rangle & \langle\{0.3,0.5,0.6,0.6,0.6\}\rangle \\
& \langle\{0.7,0.7,0.5,0.6,0.9\}\rangle & \langle\{0.3,0.2,0.6,0.3,0.3\}\rangle & \langle\{0.3,0.4,0.6,0.7,0.7\}\rangle \\
& \langle\{0.7,0.8,0.5,0.5,0.9\}\rangle & \langle\{0.3,0.4,0.4,0.6,0.8\}\rangle & \langle\{0.8,0.8,0.8,0.9,0.9\}\rangle \\
& \langle\{0.7,0.6,0.5,0.6,0.9\}\rangle & \langle\{0.3,0.3,0.5,0.6,0.6\}\rangle & \langle\{0.6,0.7,0.8,0.6,0.9\}\rangle \\
& \langle\{0.8,0.7,0.5,0.5,0.6\}\rangle & \langle\{0.1,0.5,0.4,0.5,0.9\}\rangle & \langle\{0.7,0.3,0.3,0.4,0.5\}\rangle \\
& \langle\{0.1,0.2,0.2,0.2,0.2\}\rangle & \langle\{0.1,0.2,0.1,0.3,0.3\}\rangle & \langle\{0.3,0.1,0.2,0.2,0.1\}\rangle \\
& \langle\{0.3,0.4,0.5,0.5,0.2\}\rangle & \langle\{0.1,0.7,0.6,0.8,0.9\}\rangle & \langle\{0.5,0.3,0.7,0.4,0.5\}\rangle \\
& \langle\{0.3,0.5,0.5,0.6,0.9\}\rangle & \langle\{0.7,0.7,0.5,0.6,0.8\}\rangle & \langle\{0.3,0.5,0.6,0.4,0.4\}\rangle
\end{array}\right) .
\end{aligned}
$$

Now, by implementing the steps of the proposed TOPSIS-CI on to this modified information, we get the final ranking order of the numbers as $\mathscr{A}_{2}>\mathscr{A}_{6}>\mathscr{A}_{3}>\mathscr{A}_{7}>\mathscr{A}_{4}>\mathscr{A}_{1}>\mathscr{A}_{5}$.

A comparison of this ranking with the previous rankings based on decision matrix $D$ shows the best and worst alternatives in direct sum, simple additive weighting, and TOPSIS-CI methods demontized to the worst and the best alternatives in TOPSIS-CI method with hesitant decision matrix $\widetilde{H}$, respectively. This means that experts believe that the documents uploaded by the 5 th organization $\mathscr{A}_{5}$ are very different from its performance in the real world. This type of evaluation will make the evaluators more careful in the selfassessment process and avoid uploading baseless documents.
As we can see from the above ranking orders of alternatives, virtual alternative $\mathscr{A}_{5}$ is the best and $\mathscr{A}_{2}$ is the worst alternative, when the decision matrix $D$ is used. While, in the DMs, as in the decision matrix $\widetilde{H}$, it is believed that $\mathscr{A}_{2}$ is the best and $\mathscr{A}_{5}$ is the worst alternatives. However, the neglect of certain special conditions has caused some functional shortcomings of an organization, which may be due to the lack of preparation by other organizations, only to be aware of that particular device, and therefore $\mathscr{A}_{2}$ will have the best evaluation. Comparing these with what was obtained by the proposed method in this paper shows that the virtual option $\mathscr{A}_{5}$, similar to decision makers, is still the worst option. But, the best option for this method is different from all other methods, which is closer to reality by looking again at the values of the HFNs matrix $\widetilde{H D}$. 


\section{Conclusion}

This paper aspires to present a novel MADM method to solve the decision-making problems under the hesitant fuzzy number environment. In the present paper, an uncertainty which is presented in the data is handled with the help of the hesitant fuzzy information which consists of two parts: real and membership parts. Keeping the advantages of it, this paper centers around to examine the issues of how to order the different numbers, by utilizing the uncertain and fuzzy information. For it, we have defined the new method named as TOPSIS-CI and studied their features. In this approach, with the support of the CI operator, all the rating information measures of the attributed are aggregated. The major benefit of using CI during the process is that it practices fuzzy measures during its computations, which not only counts all the parameters but also reflects the interaction between all becoming pairs of the attributes. Finally, a TOPSIS method has been introduced with the stated distance measures and by consideration of the multiexperts. In it, we aggregate the rating of the alternatives by using CI and distance measures and hence compute the positive and negative ideals. Lastly, we established an approach, TOPSISCI, for addressing the fuzzy decision-making issue about assessment of the information. The advantage of the proposed method is that it avoids the wrong decision based on the small discrimination. A numerical illustration is given to describe the approach, and a discussion is given to determine the functionality as well as the supremacy of the MADM method. From the investigation, it is inferred that the given MADM is more stable and sustains to determine the problems under the diverse fuzzy environment.

In the future, there is a scope of elongating the research to some uncertain environment. Also, in the present work, nonmembership degrees have not be considered during the evaluations. These drawbacks will be studied in our future work. Also, we will define some more generalized algorithms to solve more complex problems such as brain hemorrhage, healthcare, nonlinear systems, and control systems to solve the complex decision-making problems [50-52].

\section{Data Availability}

No data were used to support this study.

\section{Conflicts of Interest}

The authors declare that they have no conflicts of interest.

\section{References}

[1] L. A. Zadeh, "Fuzzy sets," Information and Control, vol. 8, no. 3, pp. 338-353, 1965.

[2] R. Bellman and L. Zadeh, "Decision-making in a fuzzy environment," Management Science, vol. 17, no. 4, pp. 141-164, 1970.

[3] S. Faizi, T. Rashid, W. Sałabun, S. Zafar, and J. Wątróbski, "Decision making with uncertainty using hesitant fuzzy sets," International Journal of Fuzzy Systems, vol. 20, no. 1, pp. 93-103, 2018.
[4] V. Torra, "Hesitant fuzzy sets," International Journal of Intelligent Systems, vol. 25, no. 6, pp. 529-539, 2010.

[5] X. Wang and E. Triantaphyllou, "Ranking irregularities when evaluating alternatives by using some ELECTRE methods," Omega, vol. 36, no. 1, pp. 45-63, 2008.

[6] W. Liu and H. Liao, "A bibliometric analysis of fuzzy decision research during 1970-2015," International Journal of Fuzzy Systems, vol. 19, no. 1, pp. 1-14, 2017.

[7] Z. Xu and M. Xia, "On distance and correlation measures of hesitant fuzzy information," International Journal of Intelligent Systems, vol. 26, no. 5, pp. 410-425, 2011.

[8] V. Torra and Y. Narukawa, "On hesistant fuzzy sets and decision," in Proceedings of the 8th IEEE International Conference on Fuzzy Systems, pp. 1378-1382, Jeju Island, South Korea, August 2009.

[9] K. T. Atanassov, "Intuitionistic fuzzy sets," Fuzzy Sets and Systems, vol. 20, no. 1, pp. 87-96, 1986.

[10] D. Deepak, B. Mathew, S. J. John, and H. Garg, "A topological structure involving hesitant fuzzy sets," Journal of Intelligent \& Fuzzy Systems, vol. 36, no. 6, pp. 6401-6412, 2019.

[11] Z. Xu and M. Xia, "Distance and similarity measures for hesitant fuzzy sets," Information Sciences, vol. 181, no. 11, pp. 2128-2138, 2011.

[12] R. Verma and B. D. Sharma, "New operations over hesitant fuzzy sets," Fuzzy Information and Engineering, vol. 5, no. 2, pp. 129-146, 2013.

[13] H. Liao, Z. Xu, and M. Xia, "Multiplicative consistency of hesitant fuzzy preference relation and its application in group decision making," International Journal of Information Technology \& Decision Making, vol. 13, no. 1, pp. 47-76, 2014.

[14] B. Farhadinia, "A series of score functions for hesitant fuzzy sets," Information Sciences, vol. 277, pp. 102-110, 2014.

[15] H. Liao, X. Wu, A. Keikha, and A. Hafezalkotob, "Power average-based score function and extension rule of hesitant fuzzy set and the hesitant power average operators," Journal of Intelligent \& Fuzzy Systems, vol. 35, no. 3, pp. 3873-3882, 2018.

[16] Z. Zhang, "Hesitant fuzzy power aggregation operators and their application to multiple attribute group decision making," Information Sciences, vol. 234, pp. 150-181, 2013.

[17] S. Lalotra and S. Singh, "Knowledge measure of hesitant fuzzy set and its application in multi-attribute decision-making," Computational and Applied Mathematics, vol. 39, p. 86, 2020.

[18] N. Zhang and G. Wei, "Extension of VIKOR method for decision making problem based on hesitant fuzzy set," Applied Mathematical Modelling, vol. 37, no. 7, pp. 4938-4947, 2013.

[19] Z. Xu and X. Zhang, "Hesitant fuzzy multi-attribute decision making based on TOPSIS with incomplete weight information," Knowledge-Based Systems, vol. 52, pp. 53-64, 2013.

[20] X. Tong and L. Yu, "MADM based on distance and correlation coefficient measures with decision-maker preferences under a hesitant fuzzy environment," Soft Computing, vol. 20, no. 11, pp. 4449-4461, 2016.

[21] Z. Xu and M. Xia, "Hesitant fuzzy entropy and cross-entropy and their use in multiattribute decision-making," International Journal of Intelligent Systems, vol. 27, no. 9, pp. 799822, 2012.

[22] M. Akram, H. Garg, and K. Zahid, "Extensions of ELECTRE-I and TOPSIS methods for group decision-making under complex Pythagorean fuzzy environment," Iranian Journal of Fuzzy Systems, vol. 17, no. 5, pp. 147-164, 2020.

[23] K. Askarifar, Z. Motaffef, and S. Aazaami, "An investment development framework in Iran's seashores using TOPSIS 
and best-worst multi-criteria decision making methods," Decision Science Letters, vol. 7, no. 1, pp. 55-64, 2018.

[24] R. Saikia, H. Garg, and P. Dutta, "Fuzzy multi-criteria decision making algorithm under intuitionistic Hesitant fuzzy set with novel distance measure," International Journal of Mathematical, Engineering and Management Sciences, vol. 5, no. 3, pp. 473-487, 2020

[25] I. Deli, "A TOPSIS method by using generalized trapezoidal hesitant fuzzy numbers and application to a robot selection problem," Journal of Intelligent \& Fuzzy Systems, vol. 38, no. 1, pp. 779-793, 2020.

[26] D. Joshi and S. Kumar, "Interval-valued intuitionistic hesitant fuzzy Choquet integral based TOPSIS method for multicriteria group decision making," European Journal of Operational Research, vol. 248, no. 1, pp. 183-191, 2016.

[27] D. Liang and Z. Xu, "The new extension of TOPSIS method for multiple criteria decision making with hesitant pythagorean fuzzy sets," Applied Soft Computing, vol. 60, pp. 167179, 2017.

[28] G. Sun, X. Guan, X. Yi, and Z. Zhou, “An innovative TOPSIS approach based on hesitant fuzzy correlation coefficient and its applications," Applied Soft Computing, vol. 68, pp. 249267, 2018.

[29] H. Garg, R. Arora, and R. Arora, "TOPSIS method based on correlation coefficient for solving decision-making problems with intuitionistic fuzzy soft set information," AIMS Mathematics, vol. 5, no. 4, p. 2944, 2020.

[30] M. Xia and Z. Xu, "Hesitant fuzzy information aggregation in decision making," International Journal of Approximate Reasoning, vol. 52, no. 3, pp. 395-407, 2011.

[31] X. Zhang and Z. Xu, "The TODIM analysis approach based on novel measured functions under hesitant fuzzy environment," Knowledge-Based Systems, vol. 61, pp. 48-58, 2014.

[32] G. Wei, "Hesitant fuzzy prioritized operators and their application to multiple attribute decision making," KnowledgeBased Systems, vol. 31, pp. 176-182, 2012.

[33] H. Liao and Z. Xu, "A VIKOR-based method for hesitant fuzzy multi-criteria decision making," Fuzzy Optimization and Decision Making, vol. 12, no. 4, pp. 373-392, 2013.

[34] X. He, "Typhoon disaster assessment based on Dombi hesitant fuzzy information aggregation operators," Natural Hazards, vol. 90, no. 3, pp. 1153-1175, 2018.

[35] G. Wei, X. Zhao, H. Wang, and R. Lin, "Hesitant fuzzy Choquet integral aggregation operators and their applications to multiple attribute decision making," Information, vol. 15, no. 2, pp. 441-448, 2012.

[36] T. Demirel, S. C. Öner, S. Tüzün, M. Deveci, M. Öner, and N. Ç. Demirel, "Choquet integral-based hesitant fuzzy decision-making to prevent soil erosion," Geoderma, vol. 313, pp. 276-289, 2018.

[37] B. Zhu, Z. Xu, and M. Xia, "Hesitant fuzzy geometric Bonferroni means," Information Sciences, vol. 205, pp. 72-85, 2012.

[38] Z. Xu and W. Zhou, "Consensus building with a group of decision makers under the hesitant probabilistic fuzzy environment," Fuzzy Optimization and Decision Making, vol. 16, no. 4, pp. 481-503, 2017.

[39] Z. Zhang and C. Wu, "Weighted hesitant fuzzy sets and their application to multi-criteria decision making," British Journal of Mathematics \& Computer Science, vol. 4, no. 8, pp. 10911123, 2014.

[40] H. Liao and Z. Xu, "Extended hesitant fuzzy hybrid weighted aggregation operators and their application in decision making," Soft Computing, vol. 19, no. 9, pp. 2551-2564, 2015.
[41] G. W. Wei, F. E. Alsaadi, T. Hayat, and A. Alsaedi, "Hesitant fuzzy linguistic arithmetic aggregation operators in multiple attribute decision making," Iranian Journal of Fuzzy Systems, vol. 13, no. 4, pp. 1-16, 2016.

[42] C. Tan, W. Yi, and X. Chen, "Hesitant fuzzy Hamacher aggregation operators for multicriteria decision making," Applied Soft Computing, vol. 26, pp. 325-349, 2015.

[43] M. Ranjbar, S. M. Miri, and S. Effati, "Hesitant fuzzy numbers with $(\alpha, k)$-cuts in compact intervals and applications," Expert Systems with Applications, vol. 151, p. 113363, 2020.

[44] C. L. Hwang and K. Yoon, Multiple Attribute Decision Making Methods and Applications A State-of-the-Art Survey, Springer, Berlin, Germany, 1981.

[45] D. Yu, Y. Wu, and W. Zhou, "Multi-criteria decision making based on choquet integral under hesitant fuzzy environment," Journal of Computational Information Systems, vol. 7, no. 12, pp. 4506-4513, 2011.

[46] H. Liao and Z. Xu, Hesitant Fuzzy Decision Making Methodologies and Applications, Springer, Berlin, Germany, 2017.

[47] M. Grabisch, T. Murofushi, and M. Sugeno, Fuzzy Measures and Integrals, Physica-Verlag, Heidelberg, Germany, 2000.

[48] G. Choquet, “Theory of capacities," Annales de L'institut Fourier, vol. 5, pp. 131-295, 1954.

[49] A. Keikha and H. Mishmast Nehi, "Fuzzified Choquet integral and its applications in MADM: a review and a new method," International Journal of Fuzzy Systems, vol. 17, no. 2, pp. 337-352, 2015.

[50] M. Deveci, U. Cali, S. Kucuksari, and N. Erdogan, "Interval type-2 fuzzy sets based multi-criteria decision-making model for offshore wind farm development in ireland," Energy, vol. 198, p. 117317, 2020.

[51] H. Garg and G. Kaur, "Quantifying gesture information in brain hemorrhage patients using probabilistic dual hesitant fuzzy sets with unknown probability information," Computers \& Industrial Engineering, vol. 140, p. 106211, 2020.

[52] H. Garg, "New ranking method for normal intuitionistic sets under crisp, interval environments and its applications to multiple attribute decision making process," Complex \& Intelligent Systems, vol. 6, no. 3, pp. 559-571, 2020. 